\title{
Ion heating resulting from pickup in magnetic reconnection exhausts
}

\author{
J. F. Drake, ${ }^{1}$ M. Swisdak, ${ }^{1}$ T. D. Phan, ${ }^{2}$ P. A. Cassak, ${ }^{3}$ M. A. Shay, ${ }^{4}$ S. T. Lepri, ${ }^{5}$ \\ R. P. Lin, ${ }^{2}$ E. Quataert, ${ }^{6}$ and T. H. Zurbuchen ${ }^{5}$ \\ Received 25 August 2008; revised 20 January 2009; accepted 19 March 2009; published 30 May 2009.
}

[1] The heating of ions downstream of the $\mathrm{x}$-line during magnetic reconnection is explored using full-particle simulations, test particle simulations, and analytic analysis. Large-scale particle simulations reveal that the ion temperature increases sharply across the boundary layer that separates the upstream plasma from the Alfvénic outflow. This boundary layer, however, does not take the form of a classical switch-off shock as discussed in the Petschek reconnection model, so the particle heating cannot be calculated from the magnetohydrodynamic, slow-shock prediction. Test particle trajectories in the fields from the simulations reveal that ions crossing the narrow boundary into the exhaust instead behave like pickup particles: they gain both a directed outflow and an effective thermal speed given by the flow speed $v_{0}$ of the exhaust. The detailed dynamics of these particles are explored by taking 1-D cuts of the simulation data across the exhaust, transforming to the deHoffman-Teller frame, and calculating explicitly the increment in the temperature, $m_{i} v_{0}^{2} / 3$, with $m_{i}$, the ion mass. We compare the model predictions with the temperature increment in solar wind exhausts measured by the ACE and Wind spacecraft, confirming that the temperature increment is proportional to the ion mass. The Wind data from 22 high-shear exhaust encounters confirm the scaling of the proton temperature increment with the square of the exhaust velocity. However, the temperature increments are consistently lower than the model prediction. Implications for understanding the production of high-energy ions in flares and the broader universe are discussed.

Citation: Drake, J. F., M. Swisdak, T. D. Phan, P. A. Cassak, M. A. Shay, S. T. Lepri, R. P. Lin, E. Quataert, and T. H. Zurbuchen (2009), Ion heating resulting from pickup in magnetic reconnection exhausts, J. Geophys. Res., 114, A05111, doi:10.1029/2008JA013701.

\section{Introduction}

[2] Magnetic reconnection is the dominant mechanism for converting stored magnetic energy in plasma systems into high-velocity flows and energetic particles. In solar flares a significant fraction of the released magnetic energy is transferred to energetic electrons and ions, with ions reaching energies in the range of $\mathrm{GeV}$, which greatly exceeds energies associated with reconnection driven Alfvénic outflows [Lin et al., 2003; Emslie et al., 2004]. The acceleration mechanism for these energetic ions remains unknown although models based on particle interaction with multiple islands in 2-D

\footnotetext{
${ }^{1}$ IREAP, University of Maryland, College Park, Maryland, USA.

${ }^{2}$ Space Sciences Laboratory, University of California, Berkeley, California, USA.

${ }^{3}$ Department of Physics, West Virginia University, Morgantown, West Virginia, USA.

${ }^{4}$ Department of Physics and Astronomy, University of Delaware, Newark, Delaware, USA.

${ }^{5}$ Department of Atmospheric, Oceanic, and Space Sciences, University of Michigan, Ann Arbor, Michigan, USA.

${ }^{6}$ Department of Astronomy, University of California, Berkeley, California, USA.
}

Copyright 2009 by the American Geophysical Union. 0148-0227/09/2008JA013701
[Matthaeus et al., 1984; Kliem, 1994; Shibata and Tanuma, 2001] and 3-D [Onofri et al., 2006] and with magnetohydrodynamic waves [Miller, 1998; Petrosian and Liu, 2004] have been proposed. In the Earth's magnetotail ions with energies in the range of hundreds of $\mathrm{keV}$ have been measured [Meng et al., 1981; Sarafopoulos et al., 2001] although unlike with electrons [Øieroset et al., 2002] the direct connection between these energetic particles and magnetic reconnection has not been firmly established.

[3] Although a causal linkage between reconnection and energetic ions within the magnetosphere has not been established, satellite crossings of the reconnection $\mathrm{x}$-line or reconnection driven outflows have clearly documented complex ion distribution functions and associated ion heating. At the magnetopause ISEE 1 and 2 data reveal ion heating associated with energetic flow events [Gosling et al., 1986]. The high-speed ion beams that characterize the plasma sheet boundary layer have been linked to reconnection in Geotail data and are interpreted as evidence for ion acceleration at the $\mathrm{x}$-line [Hoshino et al., 1998]. Alfvénic counterstreaming ions in reconnection exhausts have been documented in the magnetotail [Hoshino et al., 1998], the solar wind [Gosling et al., 2005b] and the magnetosheath [Phan et al., 2007b] and are an expected feature of 
reconnection outflows [Hoshino et al., 1998; Arzner and Scholer, 2001]. A basic question therefore is what fraction of the magnetic energy released during reconnection ends up as ion thermal motion as opposed to convective flow.

[4] In this manuscript we explore ion heating and acceleration during magnetic reconnection using large-scale particle-in-cell simulations, test particle simulations and analytic modeling. In section 2 we present the results of 2-D particle-in-cell (PIC) simulations that show that the ion temperature increases sharply across a narrow boundary layer that separates the upstream plasma from the Alfvénic exhaust. As a result, the ion heating during reconnection is dominated by the large-scale outflow exhausts rather than the x-line proper. The exhaust boundary does not take the form of a Petschek-like slow shock so the sharp temperature increments do not result from the dissipation associated with slow shocks. Alternate heating mechanisms are therefore explored. In section 3 the results of test particle simulations using the fields from the PIC simulations are presented. The trajectories reveal that the ions become demagnetized as they cross the exhaust boundary. Upon entering the exhaust, they are essentially at zero velocity within a local plasma flowing at the Alfvén speed. As a result they behave like classic pickup particles [Möbius et al., 1985] and gain an effective thermal speed equal to the exhaust velocity once they have been "picked up" by the exhaust. In section 4 the dynamics of ions as they enter the exhaust are further explored by using the fields from 1-D cuts across the exhaust. We demonstrate that the electric field in these cuts can be eliminated by transforming to the reference frame of the high-speed exhaust (the deHoffmanTeller frame). The ion motion in the resulting magnetic fields can be calculated analytically. Like a classical pickup particle, the ions gain an effective thermal velocity equal to the exhaust velocity. Predictions of the temperature increments perpendicular and parallel to the magnetic field within the exhaust are presented and compared with a Cluster encounter with a reconnection exhaust in the Earth's magnetosheath. Ions with mass greater than that of the dominant protons are also predicted to act like pickup particles, gain an Alfvénic thermal speed and therefore gain energy proportional to their mass, which is, to lowest order, a characteristic feature of thermal ions in the solar wind [von Steiger and Zurbuchen, 2006]. Finally in section 5 we compared the predictions with Wind and ACE data from encounters with reconnection exhausts in the solar wind [Gosling et al., 2005b; Phan et al., 2006; Gosling, 2007]. The Wind and ACE data confirm that the ion temperature increment in the exhaust scales with the square of the exhaust velocity and the ion mass. However, the observed temperature increments are substantially below the predictions. In section 6 we discuss the results, discrepancies with observations and implications for understanding energetic ions produced during solar flares.

\section{Simulations}

[5] Our simulations are performed with the particle-incell code p3d [Zeiler et al., 2002]. The results are presented in normalized units: the magnetic field to the asymptotic value of the reversed field, the density to the value at the center of the current sheet minus the uniform background density, velocities to the proton Alfvén speed $v_{A}$, lengths to the proton inertial length $d_{p}$, times to the inverse proton cyclotron frequency $\Omega_{c p}^{-1}$, and temperatures to $m_{p} v_{A}^{2}$. We consider a system periodic in the $x-y$ plane where flow into and away from the $\mathrm{x}$-line are parallel to $\hat{\mathbf{y}}$ and $\hat{\mathbf{x}}$, respectively. The reconnection electric field is parallel to $\hat{\mathbf{z}}$. The initial equilibrium consists of two Harris current sheets superimposed on a ambient population of uniform density. The reconnection magnetic field is given by $B_{x}=\tanh [(y-$ $\left.\left.L_{y} / 4\right) / w_{0}\right]-\tanh \left[\left(y-3 L_{y} / 4\right) / w_{0}\right]-1$, where $w_{0}=1.5$ is the half width of the initial current sheet. There is no initial outof-plane field $B_{z}$. The box lengths $L_{y}$ and $L_{x}$ in the $y$ and $x$ direction are 102.4 and 204.8, respectively. The initial density profile is the usual Harris form plus a uniform background of 0.2. The electron and ion temperatures, $T_{e}=$ $1 / 12$ and $T_{p}=5 / 12$, are initially uniform. Since ion heating and acceleration should be insensitive to the ion to electron mass ratio, we present data from a simulation with $m_{p} / m_{e}=$ 25 , which is sufficient to separate the spatial scales of the two species. The grid scale $\Delta=0.05$ and the speed of light is $c=15$ and there are 100 particles per grid cell in the lowdensity region. The simulations presented here are two dimensional, i.e., $\partial / \partial z=0$. Reconnection is initiated with a small initial magnetic perturbation that produces a single magnetic island on each current layer.

[6] In analyzing the data it is convenient to define temperatures parallel and perpendicular to the local magnetic field,

$$
\begin{gathered}
T_{\|}=\frac{1}{n} \int d \mathbf{v} m_{p}\left(v_{\|}-u_{\|}\right)^{2} f(\mathbf{x}, \mathbf{v}, t), \\
T_{\perp}=\frac{1}{2 n} \int d \mathbf{v} m_{p}\left|\mathbf{v}_{\perp}-\mathbf{u}_{\perp}\right|^{2} f(\mathbf{x}, \mathbf{v}, t),
\end{gathered}
$$

where $\mathbf{u}_{\|}$and $\mathbf{u}_{\perp}$ are the plasma velocities parallel and perpendicular to the local magnetic field. We emphasize that the distribution function $f$ within the regions of interest is generally a complicated function and not simply a Maxwellian distribution.

[7] The rate of reconnection for this simulation has been presented previously [Shay et al., 2007]. As in other simulations of the double Harris equilibrium, the rate of reconnection rises to a nearly constant value $(\sim 0.14)$ until late time when the islands on adjacent current layers overlap. In Figure 1 we show plots of the out-of-plane electron current $j_{e z}$, the in-plane magnetic field, the Hall magnetic and electric fields, $B_{z}$ and $E_{y}$, and the ion parallel and perpendicular temperatures during the steady reconnection period at $t=204.5$. At this time the results remain insensitive to the periodicity of the simulation domain [Shay et al., 2007; Drake et al., 2008]. The ion parallel and perpendicular temperatures rise sharply in the regions where the Hall fields are large, with the increase in $T_{\|}$exceeding that of $T_{\perp}$ [Krauss-Varban and Omidi, 1995; Hoshino et al., 1998]. Not shown is the exhaust velocity $v_{x}$ which peaks in the same region, driven dominantly by the Hall fields [Drake et al., 2008]. The narrow high-temperature region sandwiched within the exhaust and which peaks near the $\mathrm{x}$-line is associated with the electron dissipation region. In the present manuscript we focus on the mechanism for ion 

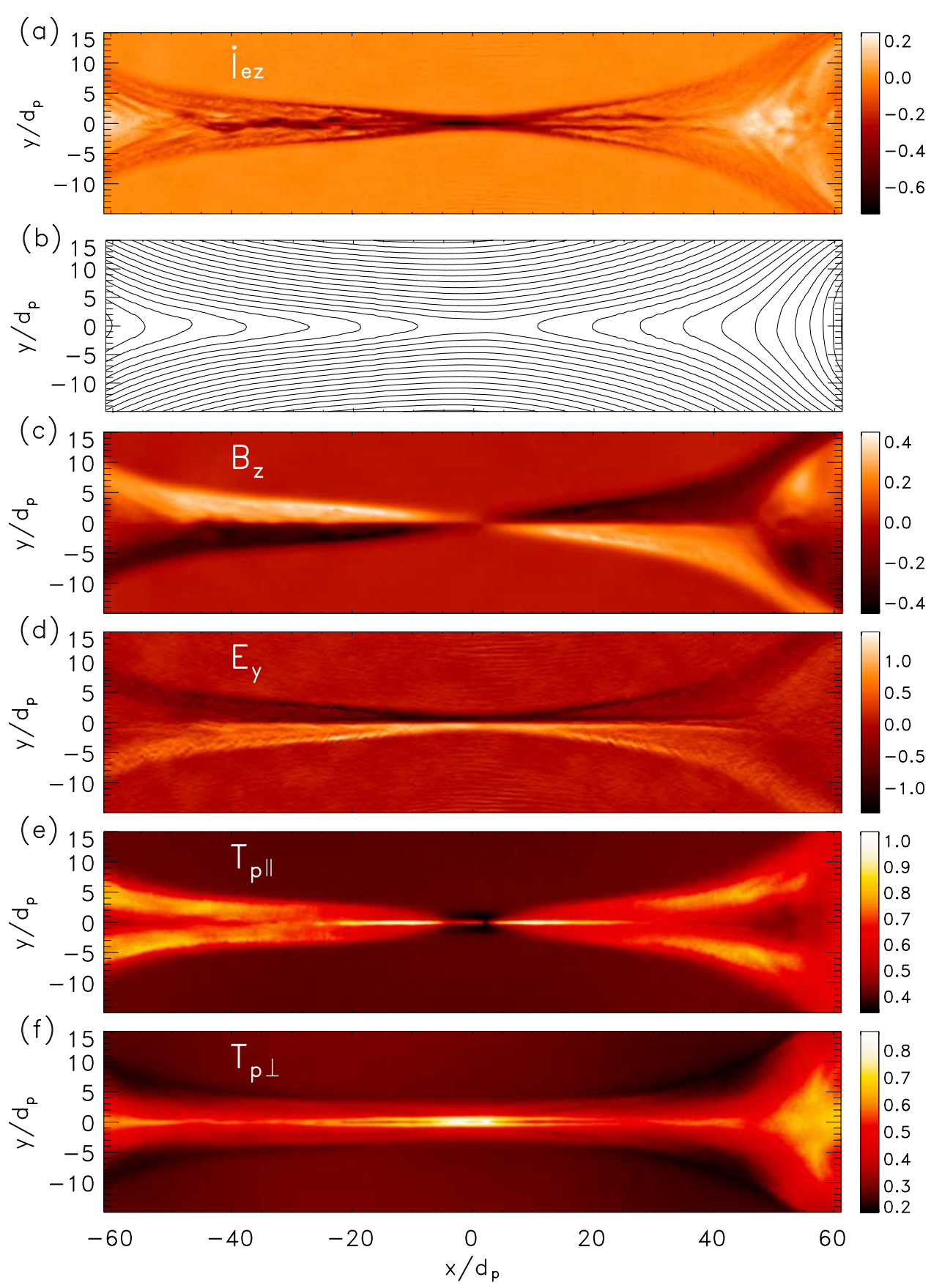

Figure 1. At late time a blowup around the $\mathrm{x}$-line of (a) the electron out-of-plane current $j_{e z} / n_{0} e c_{A}$, (b) the in-plane magnetic field lines, (c) the out-of-plane Hall magnetic field $B_{z} / B_{0}$, (d) the in-plane Hall electric field $c E_{y} / B_{0} c_{A}$, and (e) the parallel $T_{p \|} / m_{p} c_{A}{ }^{2}$ and (f) the perpendicular $T_{p \perp} / m_{p} c_{A}{ }^{2}$ proton temperatures.

heating in the larger exhaust, which because of its size dominates the ion energetics.

[8] The sharp rise in the ion parallel and perpendicular temperatures at the boundary of the exhaust might suggest that the heating is the result of a standing slow shock as discussed by Petschek in his model of reconnection [Petschek, 1964; Coroniti, 1971]. The boundary of the exhaust, however, is not a classical switch-off shock. The magnetic fields $B_{x}$ and $B_{z}$ within the exhaust are zero downstream of the switch-off shock, which is not the case in the simulation data in Figure 1. Switch-off shocks have never been documented in particle simulations of reconnection. We therefore must explore other mechanisms for the ion heating shown in Figure 1.

\section{Test Particle Trajectories in 2-D Simulation Fields}

[9] To understand the dynamics of ions in the exhaust region we have computed the orbits of test particles in the self-consistent fields of the simulation. Shown in Figure 2a is the trajectory of a proton on a background of the Hall electric 

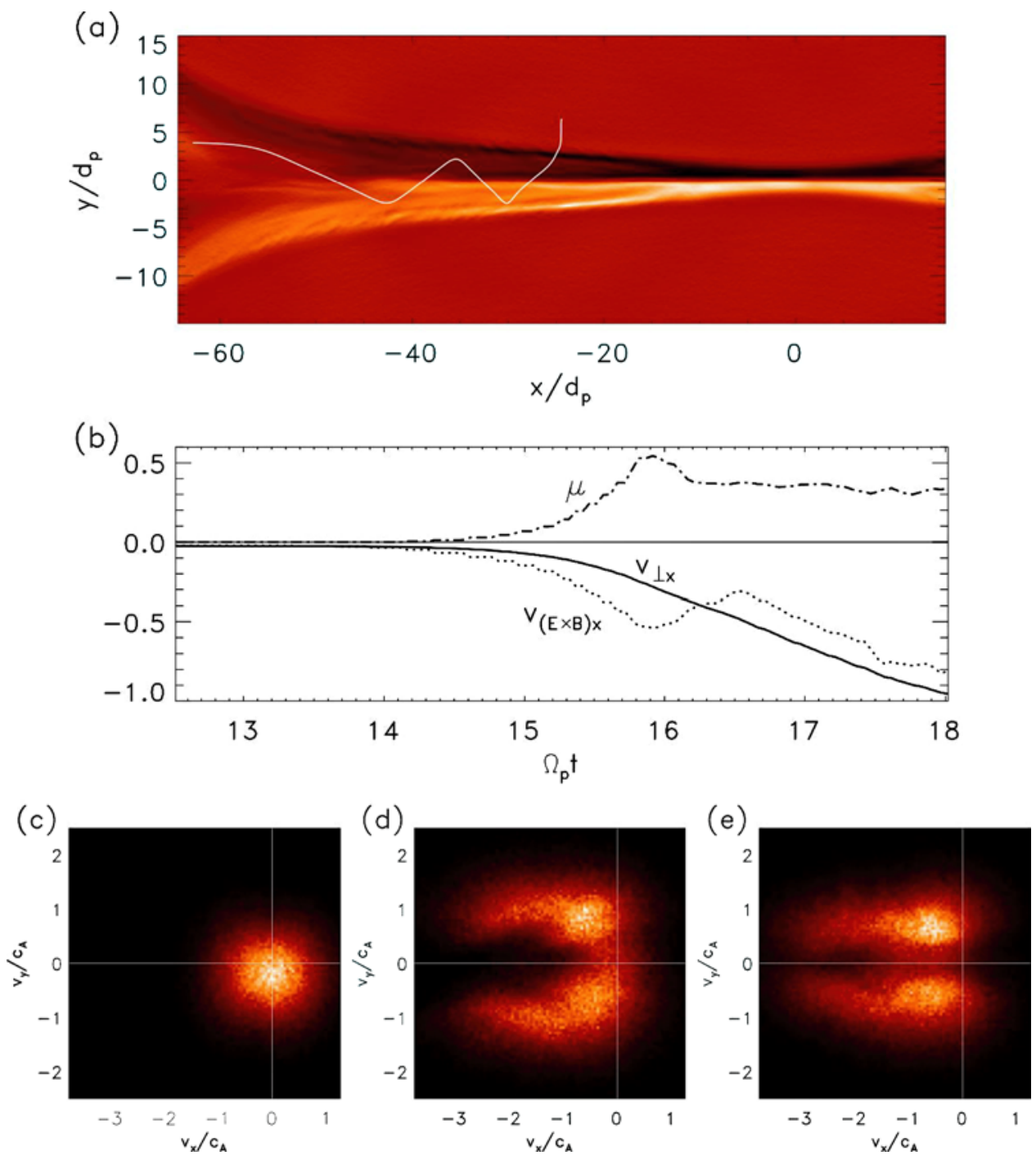

Figure 2. (a) The trajectory of a proton with a small initial thermal velocity superimposed on the Hall electric field $E_{y}$ from the simulation of Figure 1. (b) The time dependence of the $x$ components of the perpendicular velocity $v_{\perp x}$ (solid) and $\mathbf{E} \times \mathbf{B}$ velocity $v_{E \times B x}$ (dotted) and the magnetic moment $\mu$ for the trajectory in Figure 2a. From the simulation the proton velocity distributions in the $x-y$ plane (c) upstream of the exhaust at $x, y=-33.3 d_{p}, 5.6 d_{p}$ and (d) in the exhaust at the midplane at $x=-33.3 d_{p}$ and (e) further downstream at $x=-46.0 d_{p}$.

field $E_{y}$. Upon entering the exhaust the particle is accelerated by $E_{y}$ in the negative $y$ direction when $y>0$ and then in positive $y$ direction as it crosses the midplane into the region $y<0$. The Lorentz force $e v_{y} B_{z} / c$ bends the particle velocity into the outflow direction as the particle undergoes several bounces across the midplane. This behavior is similar to the ion dynamics inferred from Cluster observations of a narrow current layer produced by reconnection in the magnetotail [Wygant et al., 2005]. Evidence for the resulting counterstreaming behavior of ions undergoing this bounce motion is visible in the ion distribution functions in the $v_{x}-v_{y}$ plane in Figures $2 \mathrm{c}-2 \mathrm{e}$. Shown in Figure $2 \mathrm{c}$ is the lobe distribution at $x, y=-33.3 d_{p}, 5.6 d_{p}$, shown in Figure $2 \mathrm{~d}$ is the midplane distribution at $x, y=-33.3 d_{p}, 0.0$, and shown in Figure $2 \mathrm{e}$ is the midplane distribution further downstream at $x, y=$ $-46.0 d_{p}, 0.0$. The lobe distribution is a cold beam with a weak drift in the negative $y$ direction, reflecting the inflow toward the exhaust. There is no evidence of the leakage of higher-energy particles from the $\mathrm{x}$-line or exhaust. The midplane distribution in Figure $2 \mathrm{~d}$ exhibits the symmetric counterstreaming behavior expected from ion entry into the exhaust from above and below. The ions with small $v_{x}$ have just entered the exhaust while those with larger values of $v_{x}$ have already been accelerated downstream. Further downstream the counterstreaming of the small $v_{x}$ particles continues while the high $v_{x}$ particles have begun to thermalize. Such counterstreaming behavior has been observed earlier in simulations [Hoshino et al., 1998; Arzner and Scholer, 2001] and in distributions measured by satellites in the magnetotail [Hoshino et al., 1998; Wygant et al., 2005], in the magnetosheath [Phan et al., 2007b] and in the solar wind [Gosling et al., 2005b].

[10] To gain greater insight into the dynamics of the ions as they enter the exhaust region, we show in Figure $2 b$ more information about the time dependence of the ion motion along its trajectory in Figure 2a. Shown are the ion perpendicular 
velocity in the $x$ direction (solid) compared with the corresponding $x$ component of $\mathbf{E} \times \mathbf{B}$ drift velocity (based on the local fields at the particle position) and the magnetic moment $\mu$. A fully magnetized particle entering the exhaust would sharply turn downstream. Instead the particle in Figure 2a first moves across the exhaust in the direction of the electric field. Along the particle trajectory the increase in $v_{\perp} x$ lags the $x$ component of the $\mathbf{E} \times \mathbf{B}$ drift (Figure 2b). As a result, the magnetic moment $\mu$ is not conserved and increases. The violation of $\mu$ conservation is a consequence of the sharpness of the boundary layer: along the particle trajectory in Figure $2 \mathrm{~b}$ the $\mathbf{E} \times \mathbf{B}$ velocity rises sharply over a time interval shorter than the cyclotron period. This behavior is exactly like that of a pickup particle that is created when a neutral particle is ionized in the solar wind. The newly ionized particle first moves in the direction of the motional electric field associated with the moving field to gain the energy required to move with the solar wind velocity. In the process it gains a thermal velocity equal to the local solar wind velocity.

\section{Test Particle Trajectories in 1-D Fields}

[11] To go beyond the previous qualitative discussion, in Figures $3 \mathrm{a}$ and $3 \mathrm{~b}$ we show cuts of the magnetic fields and the electric fields across the exhaust region at $x=-36.9$. The magnetic field $B_{x}$ reverses direction across the exhaust but does not exhibit evidence of the switch-off, slow-mode shocks that are expected to bound the reconnection exhaust further downstream [Petschek, 1964; Coroniti, 1971]. The Hall field $B_{z}$ increases sharply at the exhaust boundary and reverses sign at the symmetry axis but remains large elsewhere in the exhaust, consistent with Cluster observations of reconnection in the magnetosheath [Phan et al., 2007b]. The reconnection electric field is nearly uniform, the Hall electric field $E_{y}$ is large and points toward the symmetry line while $E_{x}$ remains small.

[12] Since the exhaust and its associated fields varies only weakly with distance downstream, we can further explore the ion dynamics by shifting to a frame moving with the local magnetic field velocity (the deHoffman-Teller frame), $v_{x}=$ $-v_{0}$. In the transformed frame the electric fields are given by $\left(E_{x}, E_{y}+v_{0} B_{z} / c, E_{z}-v_{0} B_{y}\right)$. The velocity $v_{0}=c E_{z} / B_{y}$ eliminates the $\mathrm{z}$ component of the electric field. A necessary condition for a single velocity $v_{x}$ to transform away both $E_{y}$ and $E_{z}$ is $\mathbf{E} \cdot \mathbf{B}=E_{y} B_{y}+E_{z} B_{z}=0$, which is fairly well satisfied in the exhaust region. The electric fields in a frame moving with a velocity $v_{x}=-1.35$ are shown in Figure $3 \mathrm{c}$. In this frame the simulation fields are reduced to small values, confirming that during steady reconnection the reconnection electric field $E_{z}$ is the motional field of the reconnected field $B_{y}$ and the Hall electric field $E_{y}$ is the motional field of the Hall field $B_{z}$ [Arzner and Scholer, 2001]. This field line velocity is slightly below the upstream Alfvén speed, which because the upstream density is 0.2 , is around 1.9 . Neglecting the remnant electric fields in the moving frame, we can now explore the ion motion in the magnetic configuration shown in Figure 3a. A major simplification is that in this frame the particle energy is conserved.

[13] The magnetic geometry in Figure 3 is similar to the well-studied system with a reversed magnetic field and constant normal magnetic field explored by Büchner and (a)

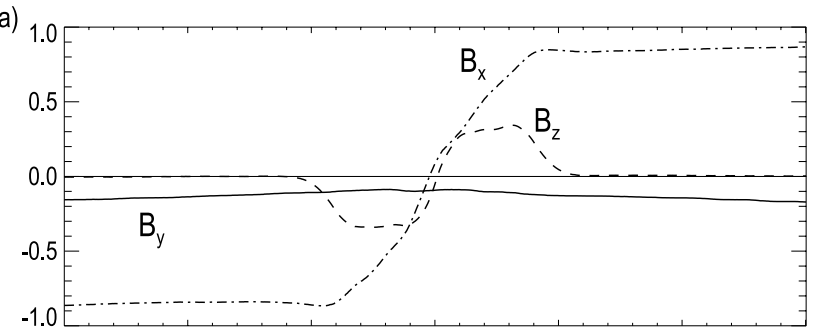

(b)

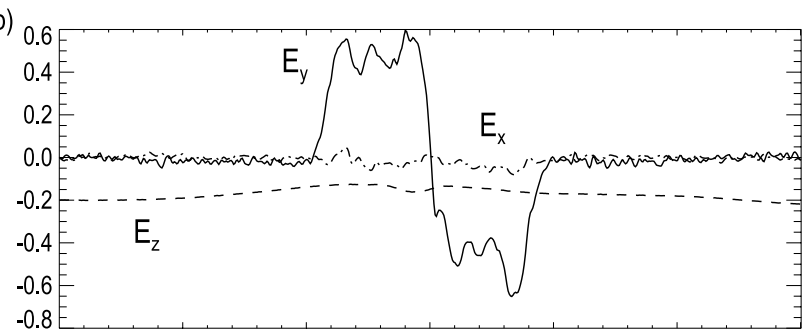

(c)

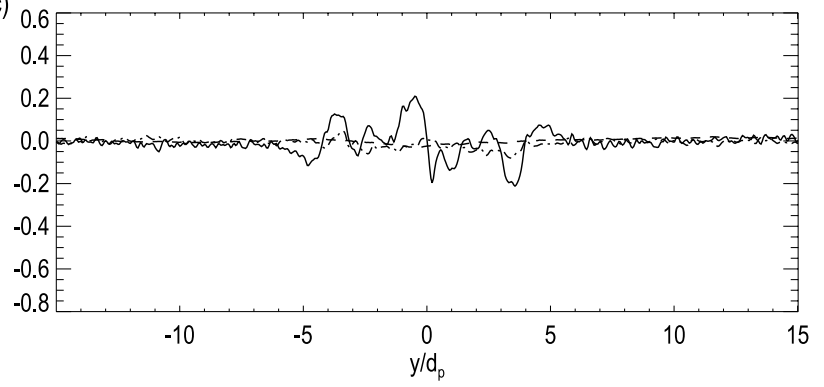

Figure 3. From the simulation shown in Figure 1 cuts of (a) the magnetic field and (b) the electric field through the reconnection exhaust at $x=-36.9 d_{p}$. (c) Cuts of the electric field at the same location in a frame moving with an instantaneous velocity $v_{x}=-1.35 c_{A}$. In all plots the $x, y$, and $z$ components are the dash-dotted, solid, and dashed lines, respectively.

Zelenyi (BZ) [Büchner and Zelenyi, 1986; Chen and Palmadesso, 1986; Büchner and Zelenyi, 1989]. In that system the particle dynamics depends on the parameter $\kappa=$ $\Omega_{y} / \omega_{b}$, the ratio of the gyration frequency $\Omega_{y}=e B_{y} / m_{p} c$ in the normal magnetic field $B_{y}$ to the bounce frequency $\omega_{b}=$ $\sqrt{v_{0} \Omega_{0 x} / L}$ in the reversed magnetic field $B_{x}$ of particles with characteristic velocity $v_{0}$, where $\Omega_{0 x}=e B_{0 x} / m_{p} c$, $d B_{x} / d y=B_{0 x} / L$ defines the scale length $L$ and the field amplitude $B_{0 x}$. Particle motion is adiabatic where $\kappa$ is large (fully magnetized particles), nearly adiabatic when $\kappa$ is small (Speiser orbit limit [Speiser, 1965]) and is chaotic in the range of $\kappa \sim 1$.

[14] The simulation fields in Figure 3 a differ from the configuration of $\mathrm{BZ}$ because of the Hall field $B_{z}$ and specifically the sharp jump in $B_{z}$ at the edge of the exhaust and its reversal across the symmetry line. For convenience in varying parameters to explore the particle dynamics, we consider a simple analytic field model with

$$
\begin{aligned}
B_{x}= & B_{0 x} \tanh (y / L) \\
B_{z}= & B_{0 z}\left[-\tanh \left((y+2 L) / L_{H}\right) / 2+\tanh \left(y / L_{H}\right)\right. \\
& \left.-\tanh \left((y-2 L) / L_{H}\right) / 2\right]
\end{aligned}
$$




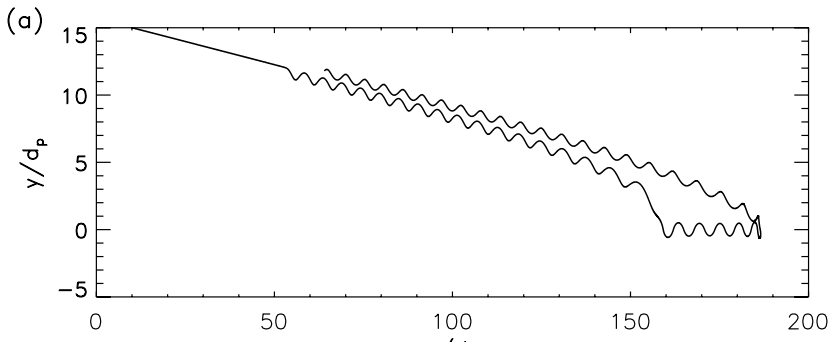

(b)

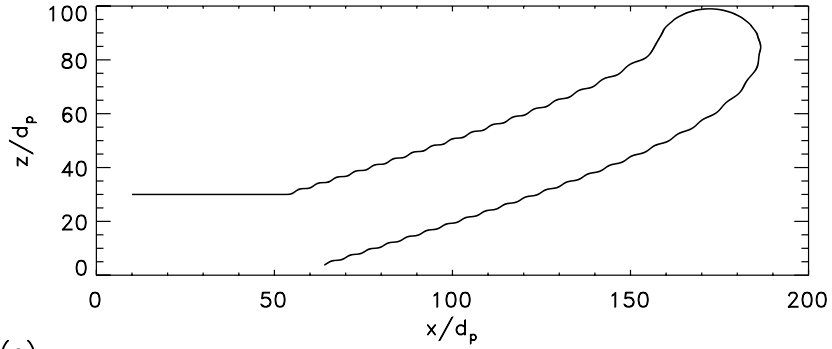

(c)

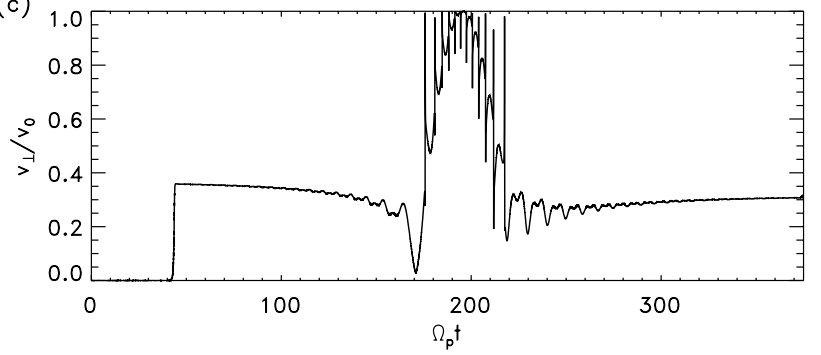

Figure 4. The dynamics of a particle in 1-D model magnetic fields of equation (3). (a and b) The trajectories in the $x-y$ and $x-z$ planes. (c) The perpendicular velocity versus time.

and $B_{0 y}$ a constant. The scale $L$ defines the width of the exhaust and the sharp jumps in $B_{z}$ at the boundaries of the exhaust and across the midplane occur over the scale length $L_{H}$, which is smaller than $d_{p}$ and therefore for any ion effectively defines a discontinuity.

[15] In Figure 4 we show the behavior of a proton with an initial parallel velocity $v_{0} / c_{A}=1.0$ in this model field with parameters given by the Cluster magnetosheath reconnection event $\left(B_{0 x}=40.0 \mathrm{nT}, B_{0 y}=3 \mathrm{nT}, B_{0 z}=18 \mathrm{nT}, L / d_{p}=\right.$ 6.0 and $L_{H} / d_{p}=0.2$ ) [Phan et al., 2007a]. In Figure 4a the particle moves toward the reversal region and suffers a jump in its perpendicular velocity $v_{\perp}$ (Figure $4 \mathrm{c}$ ) and therefore $\mu$ as it enters the exhaust. Its motion gradually turns toward the $z$ direction as $B_{x}$ decreases compared with $B_{z}$. The particle then enters the reversal region and undergoes rapid bounce motion across the midplane in a Speiser-like orbit. Its $z$ component of velocity gradually rotates into the negative $z$ direction and the particle then escapes. For large values of $L / d_{p}$ such as in this example the dynamics in the reversal region do not further increase $v_{\perp}$.

[16] An important question is whether it is $E_{z}$ or $E_{y}$ that is responsible for the energy gain of the ions accelerated in the exhaust. It was previously suggested that it was the reflection from the Hall electric field $E_{y}$ [Wygant et al., 2005]. In the trajectory in Figure 4 the ion has a net displacement in the $z$ direction. In the $\mathrm{x}$-line frame this displacement is unchanged and enables the particle to gain energy from the reconnection electric field. The Hall electric field $E_{y}$ arises from a potential. During the oscillatory ion motion along $y$ the ions gain and then return energy to this field and it therefore does not contribute net energy to the ions. Near the x-line the electric field $E_{x}$ accelerates the ions in the outflow direction [Drake et al., 2008] but this field is small in the downstream region (Figure 3 ).

[17] The jump in $v_{\perp}$ at the exhaust boundary can be calculated analytically. Upstream of the exhaust the ion velocity is parallel to $\mathbf{B}$ and approximately given by the deHoffman-Teller velocity $v_{0}$ if the initial thermal spread is neglected and $B_{0 y} \ll B_{0 x}$. Thus, upstream in the transformed frame $\mathbf{v}=\left(v_{0}, v_{0} B_{0 y} / B, 0\right)$. As the particle crosses the boundary layer, the velocity components do not change. Thus, just inside of the exhaust, since the magnetic field has twisted in the $z$ direction, the ions will have both parallel and perpendicular components. Neglecting corrections of order $B_{0 y}^{2} / B^{2}$, we find

$$
v_{\|}=v_{0} B_{0 x} / B_{0} ; \quad v_{\perp}=v_{0} B_{0 z} / B_{0}
$$

where $B_{0}^{2}=B_{0 x}^{2}+B_{0 z}^{2}$. Within the exhaust particles remain adiabatic until they reach the region of field reversal (the decrease in $v_{\perp}$ between $\Omega_{p} t=50$ and $\Omega_{p} t=160$ in Figure 4 is consistent with the conservation of $\mu$ ). As in the BZ model, in the absence of $B_{0 y}$ the particle motion is regular and particles bounce across the layer with a characteristic frequency $\omega_{b H}$ given by

$$
\omega_{b H}^{2}=\frac{1}{2} \frac{\omega_{b}^{4}}{\sqrt{\Omega_{0 z}^{4}+\omega_{b}^{4}}-\Omega_{0 z}^{2}},
$$

with $\Omega_{0 z}=e B_{0 z} / m_{i} c$ the gyro frequency in the Hall field $B_{0 z}$. In the limits of large and small $\omega_{b} / \Omega_{0 z}, \omega_{b H}$ is given by $\omega_{b}$ and $\Omega_{0 z}$, respectively. For the Cluster event $\omega_{b}=1.6 / \mathrm{s}, \Omega_{0 z}=1.7 / \mathrm{s}$, $\omega_{b H}=1.8 / \mathrm{s}$ and $\Omega_{0 y}=0.29 / \mathrm{s}$. In the BZ prescription the particle dynamics should depend on the parameter

$$
\kappa_{H}=\frac{\Omega_{0 y}}{\omega_{b H}},
$$

the ratio of the gyration frequency in the $B_{0 y}$ field $\Omega_{0 y}$ to the bounce frequency, which for the Cluster event is 0.16 . The rapid bounce motion across the reversal region compared with the slow rotation in the $B_{0 y}$ field for small $\kappa_{H}$ is the reason for the Speiser-like trajectory in Figure 4. The ratio of the three magnetic field components, $B_{x}: B_{y}: B_{z}=1.0: 0.1$ : 0.4 and the characteristic velocity $v_{0}=c_{A x}$ are the generic parameters for a system undergoing fast reconnection. The only parameter that can vary significantly from these values is therefore the scale length $L$ which can vary strongly, depending on the width of the exhaust and therefore the proximity to the x-line. The limiting value of $\kappa_{H}$ for large $L$ is simply $B_{0 y} / B_{0 z}$ which remains less than unity. Decreasing $L$ reduces $\kappa_{H}$ further so reconnection outflows typically remain in the Speiser-like regime of weakly magnetized ions. Sufficiently far downstream of the x-line where the Hall fields are no longer important, the ions may behave differently.

[18] In Figure 4 the ion exits the reversal region with $v_{\perp}$ comparable to its value on entry. This result is generic for 
small $\kappa_{H}$ and can be demonstrated analytically (B. Brown et al., private communication, 2008). In the 1-D model the ions can apparently interact a second time with the exhaust boundary (at the end of the trajectory in Figure 4). This second interaction is an artifact of the 1-D model and is not possible in the original 2-D system. In the region where the Hall fields have significant amplitude, the exhaust boundary closely maps the magnetic separatrix because of the rapid propagation of the kinetic Alfvén wave, which carries the Hall fields downstream [Drake et al., 2008]. Once an ion crosses the separatrix, the reconnection electric field continues to carry the ion downstream of the separatrix and it cannot interact with the separatrix a second time. In the frame of reference of the Alfvénic outflow the exhaust boundary propagates away from the reversal region with a velocity $v_{y}=v_{0} B_{0 y} / B_{x}$, which exceeds the ion velocity in this direction since the ion parallel velocity is limited by $v_{0}$. There is therefore no leakage of heated ions upstream of the exhaust boundary, consistent with Figure 4c.

[19] To complete the velocity calculation we take the parallel and perpendicular velocities in equation (4) after the particle is ejected from the reversal region and transform back to the frame of the $\mathrm{x}$-line, which yields $\mathbf{v}=$ $2.0 v_{\|} \mathbf{b}+\mathbf{v}_{\perp}+\mathbf{v}_{E \times B}$, where $\mathbf{b}=\mathbf{B} / B$ and $\mathbf{v}_{E \times B}$ is the $\mathbf{E} \times \mathbf{B}$ drift velocity. This result differs from the well-known acceleration of ions in a 1-D current sheet [Cowley and Shull, 1983] because of the nonadiabatic behavior at the exhaust boundary (see Figures 2 and 4). The reflected particles interpenetrate with particles that have already crossed the boundary of the exhaust but have not passed through the reversal region. The resulting effective parallel and perpendicular temperatures are

$$
T_{\|}=m_{i} v_{0}^{2} \frac{B_{0 x}^{2}}{B_{0}^{2}} \quad T_{\perp}=\frac{1}{2} m_{i} v_{0}^{2} \frac{B_{0 z}^{2}}{B_{0}^{2}}
$$

with a total temperature

$$
T=\left(T_{\|}+2 T_{\perp}\right) / 3=\frac{1}{3} m_{i} v_{0}^{2}
$$

with $m_{i}$ the ion mass, which need not be equal to the proton mass. We note that a similar expression for $T$ has been proposed to describe the proton temperature increase in hybrid simulations of reconnection [Krauss-Varban and Welsch, 2007]. We can compare the predicted increase in $T$, $T_{\|}$and $T_{\perp}$ with that measured in the simulations of Figure 1. The increase in the total temperature $\Delta T$ is easiest to compare since it is relatively constant across the exhaust. On the basis of the exhaust velocity of $1.35 c_{A}, \Delta T \simeq$ $0.6 m_{p} c_{A}^{2}$, close to the measured value of $0.5 m_{p} c_{A}^{2}$. The increases, $\Delta T_{\|}$and $\Delta T_{\perp}$, are more complex because they vary across the exhaust. The expected increments are $\Delta T_{\|}=$ $1.6 m_{p} c_{A}^{2}$ and $\Delta T_{\perp}=0.11 m_{p} c_{A}^{2}$ compared to the measured values $\Delta T_{\|}=0.8 m_{p} c_{A}^{2}$ and $\Delta T_{\perp}=(0.2-0.6) m_{p} c_{A}^{2}$ in the simulation. The predicted increase of $T_{\|}$is well above that seen in the simulation while that of $T_{\perp}$ is well below the simulation result. The scattering of the parallel into perpendicular velocity during the Speiser bounce motion near the reversal region is clearly playing a role in the simulation. $T_{\perp}$ peaks at the midplane at $0.6 m_{p} c_{A}^{2}$ where at the same time $T_{\|}$sharply decreases (see Figures 1e, 1f, and 4). The fire hose or another instability driven by the large anisotropy in the temperature could also scatter the ions and therefore increase $T_{\perp}$ at the expense of $T_{\|}$. We see some evidence for this in time-dependent, transverse flapping of the electron out-of-plane current layer. This can be seen in the warping of $j_{e z}$ in Figure 1a downstream of the $\mathrm{x}$-line, which results from the time-dependent flapping of the exhaust. In earlier large-scale hybrid simulations an anisotropy instability was able to disrupt the entire exhaust [Karimabadi et al., 1999; Arzner and Scholer, 2001]. This is not seen in the simulations of Figure 1.

[20] Direct comparisons of the predictions of equations (7) and (8) can be made with satellite observations. In the Cluster magnetosheath reconnection event $T, T_{\|}$and $T_{\perp}$ increase in the exhaust by $135 \mathrm{eV}, 185 \mathrm{eV}$ and $110 \mathrm{eV}$, respectively [Phan et al., 2007b]. Using the measured exhaust velocity of $180 \mathrm{~km} / \mathrm{s}$ and the magnetic field parameters presented previously, the predicted increases in $T, T_{\|}$and $T_{\perp}$ are $113 \mathrm{eV}, 280 \mathrm{eV}$ and $28 \mathrm{eV}$, respectively. Thus, as in the comparison with the data from the simulation the predicted increase $T$ is close to the measured value while increase in $T_{\perp}\left(T_{\|}\right)$falls well below (above) that measured, again suggesting that wave induced scattering may be active in the exhaust.

\section{Comparison With Solar Wind Data From the Wind and ACE Spacecraft}

[21] There is now a wealth of data on crossings of reconnection exhausts in the solar wind [Gosling et al., 2005b; Phan et al., 2006; Gosling et al., 2006]. Because of the large spatial extent of the typically encountered reconnection exhausts in the solar wind, these crossings must be far downstream of the $x$-line where the magnetic structure of the exhausts is very different from that closer to the $\mathrm{x}$-line. The component of the magnetic field that reverses typically drops sharply as the spacecraft enters the exhaust as in Petschek's model [Petschek, 1964] although the validity of the switch-off, slow-shock description of this boundary layer has not been established. The presence of counterstreaming ions across the entire exhaust [Gosling et al., 2005 b] seems to suggest that there is insufficient velocity space scattering to justify the simple MHD slow-shock description. Nevertheless, the MHD slow-shock prediction of the temperature increment of protons is a useful reference. In the limit of low upstream ion $\beta$ the predicted proton density and temperature increments for switch-off, slow shocks is

$$
\Delta n=\frac{3}{2} n_{0} ; \quad \Delta T_{p}=\frac{1}{5} m_{p} c_{A}^{2},
$$

where $c_{A}$ is the upstream Alfvén speed. Thus, the slowshock model predicts a somewhat lower temperature increase than that of the pickup model (equation (8)).

[22] There remain significant uncertainties in the theoretically expected structure of the highly oblique (the angle $\theta_{B n}$ between the shock normal and the magnetic field exceeding $80^{\circ}$ ) slow shocks associated with collisionless reconnection. In the two-fluid model the shock takes the switch-off form with a trailing wave train with a wavelength of $d_{i}$ [Coroniti, 


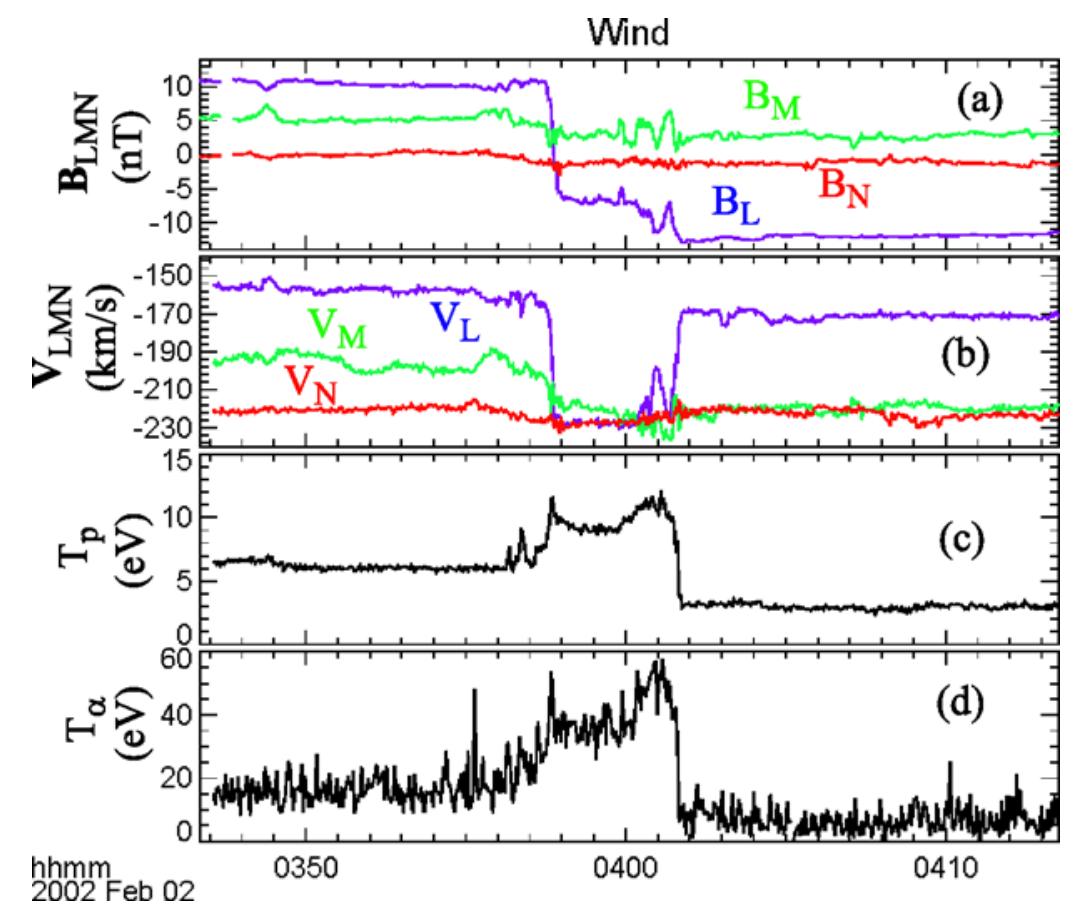

Figure 5. The time dependence of the magnetic fields $B_{L M N}$ and velocities $v_{L M N}$ in minimum variance coordinates, where the blue, green, and red lines are the L, M, and N components, respectively; the proton temperature $T_{p}$ and the alpha particle temperature $T_{\alpha}$ from a Wind spacecraft encounter with a large-scale reconnection exhaust in the solar wind [Phan et al., 2006]. Note the factor of four difference between the temperature scales.

1971]. Simulations of reconnection, however, have not yet produced the switch-off slow shocks that are expected to bound the exhaust [Arzner and Scholer, 2001]. In 1-D particle simulations of slow shocks the expected switchoff character and associated wave train develop for $\theta_{B n}<$ $80^{\circ}$ but not for $\theta_{B n}>80^{\circ}$ [Yin et al., 2005, 2007] (the slow shock does not propagate away from the simulation boundary). The reason for the failure of the highly oblique slow shocks to form remains unclear.

[23] In the frame moving with the outflow exhaust (deHoffman-Teller frame) the upstream ions again move parallel to the local magnetic field toward the exhaust. Their behavior upon crossing into the exhaust will depend on the structure of the boundary layer. If the scale length $L_{t}$ of the transition is long compared to $d_{p}$, protons will remain adiabatic at the crossing and the ions in the exhaust should be characterized by the counterstreaming Alfvénic beams predicted in previous 1-D models [Cowley and Shull, 1983]. There is support for this counterstreaming beam model in the observational data [Gosling et al., 2005b]. Even if the protons are adiabatic, higher-mass ions, because of their lower gyrofrequencies and longer inertial lengths, can transition to the pickup regime, defined by $\Omega_{i}<v_{0} / L_{t} \sim$ $c_{A} / L_{t}$ or $m_{i} / m_{p}>L_{t} / d_{p}$. In the pickup regime most of the parallel energy upstream goes into the perpendicular temperature (as a ring distribution): the ion, moving along $\mathbf{B}$ upstream, finds itself moving across $\mathbf{B}$ in the exhaust because of the sharp rotation of the field at the boundary. Thus, evidence of $T_{i \perp}>T_{i \|}$ while $T_{p \|}>T_{p \perp}$ would support this picture. If the exhaust boundaries take the form of switch-off, slow shocks, significant scattering of incoming parallel-streaming protons will take place at the shock transition either because of nonadiabatic behavior (in the $d_{p}$ scale structure of the shock transition) or as a result of the interaction with locally generated turbulence [Yin et al., 2005, 2007]. Higher-mass ions are again likely to see the transition as a discontinuity and their downstream temperature should be characterized by $T_{i \perp}>T_{i \|}$. In either the gradual transition scenario or the abrupt transition, slowshock scenario the available free energy is from the streaming velocity of ions upstream of the exhaust so the total ion temperature gain should be given approximately by equation (8), $T_{i}=m_{i} v_{0}^{2} / 3$.

[24] Given the uncertainties of theoretical models of the structure of the boundaries of the reconnection exhaust and the resulting uncertainties of the relative magnitudes of $T_{\|}$ and $T_{\perp}$, here we seek to compare only the observed increases in the total temperature of various ion species as the Wind and ACE spacecraft enter solar wind reconnection exhausts.

[25] The encounter of the Wind, Cluster and ACE spacecraft with an extended reconnection exhaust on 2 February 2002 has been well documented [Phan et al., 2006]. The Wind spacecraft encountered the exhaust when it was located at $(9 \hat{\mathbf{x}},-321 \hat{\mathbf{y}}, 16 \hat{\mathbf{z}})$ in geocentric elliptic coordinates (GSE). In Figure 5 are plotted the time series of the three components of the magnetic field and plasma flow velocity and the proton and alpha particle temperatures. The magnetic fields and flows are plotted in minimum variance coordinates, where $L$ is along the maximum variance 


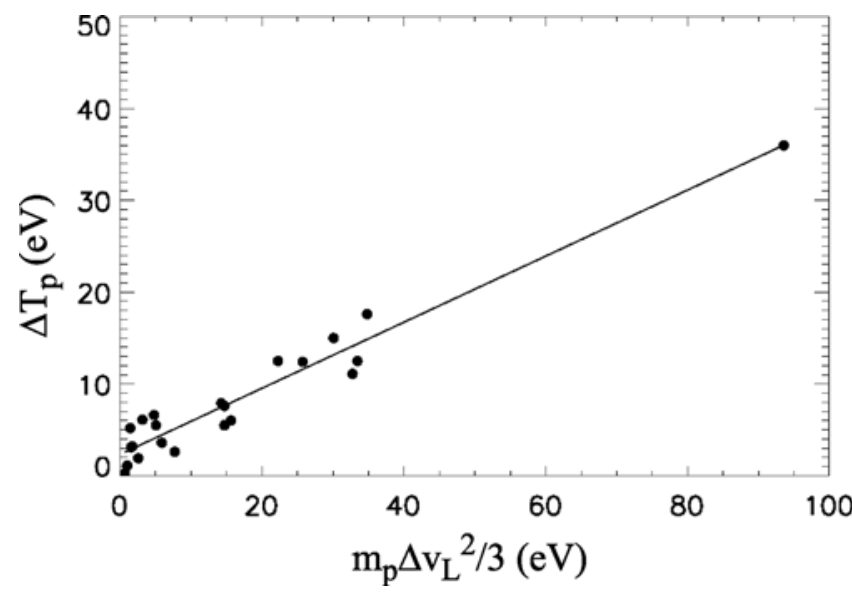

Figure 6. The temperature increment in 22 solar wind exhaust encounters with magnetic shear greater than $120^{\circ}$ versus the predicted temperature increment $m_{p} \Delta v_{L}^{2} / 3$. The line that fits through the data has a slope of 0.39 .

direction (outflow direction), $M$ is intermediate variance direction (out-of-plane direction) and $N$ is the minimum variance direction (normal to the current sheet). In terms of the coordinates of our simulations $(L, M, N)=(x, z,-y)$. The reconnecting field $B_{L}$ has nearly equal magnitude on either side of the current sheet. The guide field $B_{M}$ was around $35 \%$ of the reversed field. The jump in the normal inflow velocity $v_{N}$ across the current sheet of around $5 \mathrm{~km} / \mathrm{s}$ corresponds to a reconnection inflow velocity of $2.5 \mathrm{~km} / \mathrm{s}$, which is $3.3 \%$ of the upstream Alfvén speed. The jump $\Delta v_{L}$ in the velocity inside of the exhaust is around $65 \mathrm{~km} / \mathrm{s}$ (as the spacecraft exits the exhaust and is slightly higher on entry). On the basis of this outflow velocity, the expected increase in the temperature based on equation (8) is $14.7 \mathrm{eV}$ for protons and four times larger or $58.6 \mathrm{eV}$ for the alphas. In Figures $5 \mathrm{c}$ and $5 \mathrm{~d}$ the proton and alpha temperatures jump sharply within the exhaust with increments of $5.5 \mathrm{eV}$ and $20 \mathrm{eV}$, respectively, which are around $40 \%$ of the predicted values. The ratio of the increment in the alpha temperature to that of the protons is close to four, the alpha to proton mass ratio, as expected.

[26] There are now a large number of documented encounters of reconnection exhausts by the Wind spacecraft. We have identified 22 of these events in which the angle between the magnetic fields on either side of the exhaust (the shear angle) is greater than $120^{\circ}$, the weak guide field limit being discussed here. In Figure 6 we plot the increment of the proton temperature $\Delta T_{p}$ versus the expected increment, $m_{p} \Delta v_{L}^{2} / 3$, for these 22 events. The straight line in Figure 6 falls though the data, indicating a linear relation between the predicted and observed temperature increments. Thus, the temperature jump scales with $\Delta v_{L}^{2}$. However, the slope of the line, 0.39 is less than unity so the temperature increases in the reconnection exhausts are smaller than expected. The temperature increment is also smaller than would be predicted by the MHD slow-shock model.

[27] Direct measurement in the solar wind of the temperature increment of various ion species can provide an important test of the mass scaling of the model. Because a longer time is needed to resolve the higher-mass ions, which are much less abundant than protons, such a comparison requires a long-duration exhaust encounter. On 1 September 2001, the ACE spacecraft encountered such an reconnection exhaust during a crossing of the heliospheric current sheet [Gosling et al., 2007]. In Figures $7 \mathrm{a}-7 \mathrm{c}$ we show the proton velocity from SWEPAM in minimum variance coordinates, where $L=(-0.78 \hat{x}, 0.60 \hat{y}, 0.14 \hat{z}), M=(0.53 \hat{x}, 0.52 \hat{y}, 0.67 \hat{z})$ and $N=(0.33 \hat{x}, 0.60 \hat{y},-0.73 \hat{z})$ in GSE coordinates. Figures $7 \mathrm{~d}$ and $7 \mathrm{e}$ show the magnetic field measured by MAG in the LMN and GSE coordinate systems. The spacecraft enters the exhaust during three time intervals marked by the vertical dashed lines. During the first two entries $V_{L}$ increases sharply and $B_{L}$ drops but does not reverse direction. The third encounter is a complete crossing of the exhaust over a period of around $1.5 \mathrm{~h}$, a duration that is sufficient for measuring the temperature increment of the high-mass ions using the Solar Wind Ion Mass Spectrometer (SWICS). In Figure $7 f$ we show the behavior of the radial ( $-\hat{x}$ in GSE coordinates) temperature $T_{r r}$ of $\mathrm{He}^{2+}, \mathrm{C}^{6+}$ and $\mathrm{O}^{6+}$, as measured by SWICS (SWICS does not give directional information). Temperature enhancements are evident during all three encounters with the exhaust. The heavy ion data has a time resolution of $12 \mathrm{~min}$, the highest resolution available from SWICS. This resolution is marginally able to resolve the temperature enhancements in the first two encounters (there is no data centered within the exhaust during the first encounter) but well resolves the temperature increments during the full crossing.

[28] Because there is no data centered on the first ACE exhaust encounter, we do not discuss this encounter further. The data from the second and third encounters are shown in Table 1. Included is the data for the three mass species, the proton exhaust velocity $\Delta v_{p L}$, the predicted temperature increment per nucleon $\Delta T_{t h}$ and the observed temperature increment per nucleon $\Delta T_{o b s}$, where per nucleon indicates that we have divided the temperature by the total number of protons and neutrons. According to the model, the energy gain per nucleon should be the same for each of the ions for each exhaust encounter. For the second (short) encounter the temperature increments are calculated with respect to the adjacent data outside of the exhaust. For this encounter the temperature increments per nucleon are, especially for the higher-mass ions, reasonably close to the prediction. For the longer third encounter the temperature increments are consistently well below the predicted values although the relative increments of the different ions are in fairly good agreement (especially those of $\mathrm{C}^{6+}$ and $\mathrm{O}^{6+}$ ). This agreement, however, is not as compelling in the data of Figure 7. The $\mathrm{C}^{6+}$ and $\mathrm{O}^{6+}$ temperatures do not track each other well inside of the exhaust. The small temperature increments in exhaust encounter 3 compared with 2 may result from the fact that SWICS is measuring mostly $T_{\|}$ in encounter 2 and mostly $T_{\perp}$ in encounter 3 , and on the basis of proton and alpha particle data the increase in $T_{\|}$is typically substantially larger than $T_{\perp}$. That this is the case can be seen in the magnetic field data in GSE coordinates in Figure 7e. In encounter $2 B_{x}$ is substantially larger than the other components so $T_{r r}$ is approximately the parallel temperature while in encounter 3 the other components of $\mathbf{B}$ 


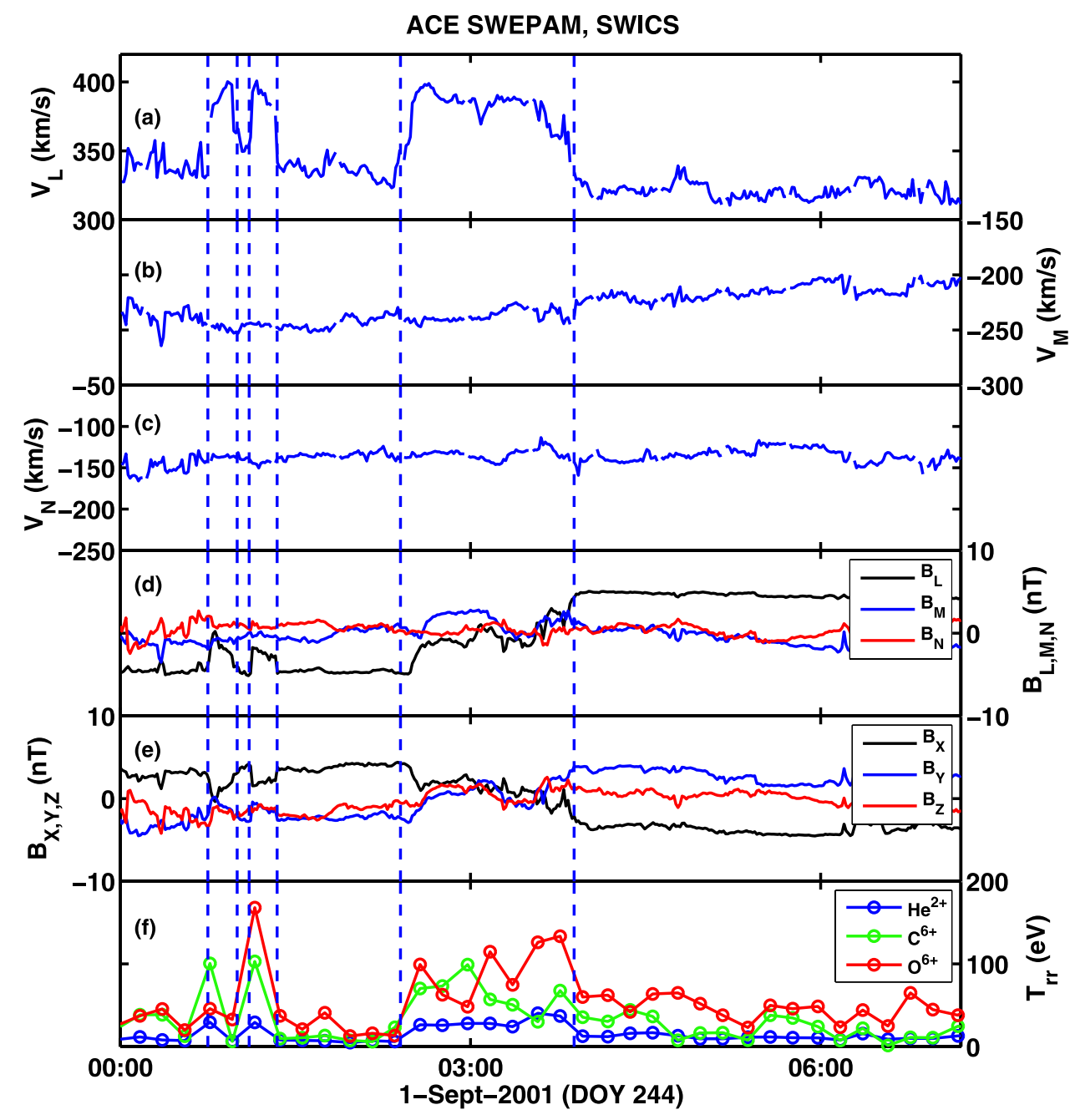

Figure 7. The time dependence of the velocities $v_{L M N}$ and magnetic fields $B_{L M N}$ in minimum variance coordinates; the magnetic fields in GSE coordinates; and the $\mathrm{He}^{2+}, \mathrm{C}^{6+}$, and $\mathrm{O}^{6+}$ radial temperatures $T_{r r}$ from an ACE spacecraft encounter with a large-scale exhaust during reconnection in the heliospheric current sheet [Gosling, 2007].

are comparable or larger so $T_{r r}$ is a mix of the parallel and perpendicular components.

\section{Summary and Discussion}

[29] We have explored the acceleration and heating of ions as they enter magnetic reconnection exhausts. In PIC simulations the perpendicular and parallel temperatures of ions increase sharply across the narrow boundary layer that separates the Alfvénic exhaust from the upstream inflowing ions (Figures 1e and 1f). The increase in the parallel temperature typically exceeds that of the perpendicular temperature. There is no evidence in the simulations for slow-mode shocks at the exhaust boundary. The temperature increments therefore do not arise from the dissipation associated with such shocks.

[30] Test particle simulations of proton entry into the exhaust using the fields from the simulations reveal that the particles behave like pickup particles [Möbius et al., 1985] since upon entry in the high-speed exhaust they are at essentially at zero velocity (like a conventional solar wind pickup particle). The ions behave nonadiabatically (the magnetic moment $\mu$ jumps sharply) both as the particles cross the narrow $\left(\sim c / \omega_{p i}\right)$ boundary layer at the edge of the exhaust and in the region of magnetic field reversal (Figure 4). As a result, the particles execute multiple reflections across the exhaust as they gradually accelerate up to the Alfvén speed along the exhaust. This bouncing behavior is manifest in the counter-

Table 1. Data from the 1 September 2001 Encounter of ACE With Reconnection Exhausts in the Heliospheric Current Sheet ${ }^{\mathrm{a}}$

\begin{tabular}{ccccc}
\hline $\begin{array}{c}\text { Exhaust } \\
\text { Number }\end{array}$ & $\Delta v_{p L}(\mathrm{~km} / \mathrm{s})$ & Ion & $\begin{array}{c}\Delta T_{\text {th }} \\
(\mathrm{eV} / \text { nucleon })\end{array}$ & $\begin{array}{c}\Delta T_{\text {obs }} \\
(\mathrm{eV} / \text { nucleon })\end{array}$ \\
\hline 2 & 55 & $\mathrm{He}^{2+}$ & 10.5 & 5.7 \\
2 & 55 & $\mathrm{C}^{6+}$ & 10.5 & 8.1 \\
2 & 55 & $\mathrm{O}^{6+}$ & 10.5 & 8.4 \\
3 & 65 & $\mathrm{He}^{2+}$ & 14.7 & 5.9 \\
3 & 65 & $\mathrm{C}^{6+}$ & 14.7 & 4.3 \\
3 & 65 & $\mathrm{O}^{6+}$ & 14.7 & 4.6 \\
\hline
\end{tabular}

${ }^{\text {a }}$ Shown are data from the second and third encounters with the exhaust, including the proton exhaust velocity $\Delta v_{p L}$, the ion species, the predicted temperature increment per nucleon $\Delta T_{t h}$, and the measured temperature increment per nucleon $\Delta T_{o b s}$. 
streaming ion particle distributions seen at the midplane (Figures 2d and 2e) in simulations. Satellite observations [Gosling et al., 2005b; Wygant et al., 2005; Phan et al., 2007b] reveal similar counterstreaming ion distributions. We have carefully studied ion acceleration by examining cuts of the simulation electric and magnetic fields across the exhaust. By transforming to the frame of reference of the exhaust $v_{0}$ the electric fields along this cut can be almost completely eliminated (Figure 3). Thus, the large in-plane Hall electric field is simply the motional electric field of the out-of-plane Hall magnetic field and, of course, the reconnection electric field is the motional field of the reconnected magnetic field.

[31] In the local exhaust frame (deHoffman-Teller frame) the electric field is negligible, ion energy is preserved and the nonadiabatic particle motion can be more easily studied. When the ion upstream thermal speed is small compared with the exhaust velocity $\left(\beta_{i} \ll 1\right)$ the inflowing ions can be taken as a cold, field-aligned beam with the exhaust velocity $v_{0}$. At the exhaust boundary the magnetic field twists sharply into the out-of-plane direction because of the presence of the Hall field (Figure 3) and as a result the ion gains a perpendicular velocity and finite magnetic moment $\mu=m_{i} v_{0}^{2} B_{0 z}^{2} / B^{3}$ (Figure 4 ) or an effective $T_{\perp}$ given in equation (8). The ion then continues toward the region of field reversal where it undergoes Speiser-like oscillations across the midplane until it exits the reversal region. Thus, the midplane bounces in Figure 2 correspond to Speiser-like motion. The bounce motion differs from that of Speiser in that the dynamics are strongly influenced by the Hall magnetic field $B_{z}$ as well as the in-plane reversed field $B_{x}$ (Figure 4). Because of the nonadiabatic behavior of the particle motion in the reversal region $v_{\perp}$ can take on a range of values. On exiting this region, however, $v_{\perp}$ reverts to its value upstream of the reversal region. This behavior is a consequence of Speiser-like motion in the reversal region. The counterstreaming of ions flowing toward and away from the reversal region produces an effective parallel temperature $T_{\|}$that is given in equation (8). Consistent with these single particle trajectories, both the parallel and perpendicular temperatures in the simulations increase within the reconnection exhaust (Figure 1) and although each varies significantly across the exhaust the total temperature is relatively uniform. The latter is perhaps because of the constancy of the particle energy in the exhaust frame. The total ion temperature in the exhaust is calculated to be

$$
T_{i}=\frac{1}{3} m_{i} v_{0}^{2}
$$

where $v_{0}$ is the proton exhaust velocity. This expression is valid for both protons and higher-mass particles and implies that the temperature increase per nucleon should be the same for all ions.

[32] The results of the simulations and resultant analytic predictions of the ion temperature increase within the reconnection outflow exhaust have been compared with observations from the ACE, Cluster and Wind satellites. Particularly important is to validate the scaling of the temperature increment in equation (10) with the ion mass and the exhaust velocity. The ion mass scaling was tested with a Wind event [Phan et al., 2006] in which the measured ratio of the temperature increment of alpha particles to the protons $\Delta T_{\alpha} / \Delta T_{p}$ was 4.3 compared with expected value of 4.0. The predicted temperature increments of $\mathrm{He}^{2+}, \mathrm{C}^{6+}$ and $\mathrm{O}^{6+}$ were compared with a reconnection encounter by ACE [Gosling, 2007] in the heliospheric current sheet (Table 1). The measured temperature increments of $\mathrm{C}^{6+}$ and $\mathrm{O}^{6+}$ using SWICS gave the same increment per nucleon while the $\mathrm{He}^{2+}$ temperature increments per nucleon differed from those of $\mathrm{C}^{6+}$ and $\mathrm{O}^{6+}$ by $20-30 \%$. When the measured temperature (along the GSE $x$ direction) was approximately parallel to the local magnetic field, the temperature increment of the three species was around $75 \%$ of the predicted value. The measured temperature was significantly smaller than that given in equation (10) when the measured temperature was perpendicular to $\mathbf{B}$, indicating that there is possibly a significant temperature anisotropy in the heavy ions.

[33] To check the scaling of the temperature increment with the exhaust velocity we identified 22 Wind encounters with reconnection exhausts. Since the present paper is limited to the case of zero guide field, we limited the data set to magnetic shear angles greater than $120^{\circ}$. The results in Figure 6 indicate that the temperature increment scales with the square of the exhaust velocity. However, the temperature increment is consistently only $39 \%$ of the expected value.

[34] The solar wind exhaust encounters of Figure 6 typically occur far from the $\mathrm{x}$-line where the structure of the boundary of the reconnection exhaust more closely resembles Petschek's slow shock [Gosling et al., 2005b, 2006; Phan et al., 2006] than the Hall magnetic field geometry that is evident closer to the x-line [Phan et al., 2007b]. Since the structure of collisionless slow shocks in the near perpendicular geometry of reconnection exhausts is not yet understood [Yin et al., 2005, 2007], this discrepancy cannot be fully resolved in the present paper. It is clear, however, that the exhaust does not simply consist of noninteracting, interpenetrating ion beams as has been suggested earlier [Gosling et al., 2005b]. In such a nondissipative model the plasma density within the exhaust would scale like $2 n_{u p} B_{N} / B_{\text {Lup }} \ll n_{u p}$, where $n_{u p}$ and $B_{\text {Lup }}$ are the density and reconnecting magnetic field upstream, $B_{N}$ is the normal magnetic field and the factor of 2 arises from the particles from the two sides of the exhaust. The density decreases because of the spreading of the flux tubes as they enter the exhaust. On the other hand in the observations the density typically increases by a factor of two in the exhaust compared with the upstream values [Gosling et al., $2005 \mathrm{~b}$, indicating that there are strong dissipative processes taking place either within the exhaust or at its boundary. In the Hall field region this density enhancement might arise from the nonadiabatic ion motion at the boundary of the exhaust and in the region of Speiser-like behavior at the midplane. In the simulations of Figure 1 the density within the exhaust is roughly twice the upstream value.

[35] The temperature increment in equation (10) combined with the observed density increase in the exhaust are inconsistent with pressure balance. Momentum balance across the exhaust boundary yields the Walen condition for the exhaust outflow $v_{0}^{2}=B_{\text {Lup }}^{2} / 4 \pi m_{p} n_{u p}$, which when combined with equation (10) yields the exhaust pressure $P_{e x}=\left(2 n_{e x} / 3 n_{u p}\right) P_{u p}$, where we have ignored the ambient 
ion temperature. For $n_{e x} / n_{u p}>1.5$ the exhaust pressure would exceed the upstream magnetic pressure. The resulting expansion of the exhaust would lower the exhaust temperature and density, which is a possible explanation for the reduced ion temperature seen in the Wind data. A confirmation of this idea must await a fuller understanding of the slow-shock structure of reconnection exhausts.

[36] The temperature increases measured in reconnection exhausts both in the solar wind and the magnetosphere are significant compared with the ambient temperatures of ions. A single entry into the exhaust does not, however, produce the high-energy ions in the $\mathrm{MeV}$ range seen within the magnetosphere [Meng et al., 1981]. In the low- $\beta$ environment of the solar corona, however, magnetic fields of $100 \mathrm{G}$ at densities of $10 \% / \mathrm{cm}^{3}$ are expected to produce exhaust ion energies in excess of $0.1 \mathrm{MeV} /$ nucleon [Krauss-Varban and Welsch, 2007], comparable to the typical energies of the ions observed in most impulsive solar energetic particle (SEP) events. Thus for small impulsive SEP events this may be the dominant acceleration mechanism. In the largest impulsive SEP events ions are observed up to $10-100 \mathrm{MeV}$ so additional acceleration is required. For large solar flares, gamma ray observations show that ions are accelerated to energies in the range of a $\mathrm{GeV}$ so a single exhaust encounter is clearly insufficient. A model of electron acceleration based on Fermi-like reflection in contracting islands has been proposed to explain electron acceleration during flares [Drake et al., 2006]. The same mechanism is expected to act on ions if they are super-Alfvénic. The interaction with just a few reconnection exhausts might seed ions to velocities large enough for them to undergo acceleration by the same Fermi mechanism.

[37] An important observation of ion acceleration relevant to impulsive SEP events is the abundance enhancement of energetic, high-mass ions in impulsive solar flares [Mason, 2007]. The enhancement compared with coronal abundances scales as $\left(m_{i} / q_{i}\right)^{3.3}$ for ions in the energy range $320-450 \mathrm{keV} /$ nucleon, where $q_{i}$ is the ion charge. In reconnection of antiparallel magnetic fields all ions behave nonadiabatically as they enter the exhaust since their values of mass to charge are greater than that of protons. Thus, these data cannot be explained with the present model. We find, however, that during reconnection with a guide field only ions with mass to charge above a critical threshold display nonadiabatic behavior and that this threshold may be related to the abundance enhancements during impulsive flares (J. F. Drake et al., Ion pickup and heating during magnetic reconnection with a guide field, submitted to Astrophysical Journal, 2009). In observations of heavy-ion temperatures in the fast solar wind the temperature per nucleon has a modest negative scaling with mass to charge [Cranmer et al., 1999], which, curiously, is opposite to that inferred from the abundance enhancements during flares, indicating that different mechanisms may be involved with the heating of ions in the solar wind in comparison with flares.

[38] An important observation in the solar wind reconnection events is the apparent absence of evidence for the production of very energetic electrons or ions [Gosling et al., 2005a] even though the spatial extent of solar wind reconnection exhausts can exceed $2 \times 10^{6} \mathrm{~km}$, which is comparable or larger in scale than impulsive flares [Tsuneta,
1996]. In contrast why is magnetic reconnection in the corona an efficient source of energetic particles? Why are energetic electrons seen during reconnection in the magnetotail [Øieroset et al., 2002; Imada et al., 2007; Chen et al., 2008]? An obvious difference between the corona and the solar wind and magnetotail is the ambient plasma $\beta$, which is very small in the corona. This implies that the Alfvén speed greatly exceeds the thermal speed in the corona so that heating associated with a pickup process such as in equation (8) or due to a slow-mode shock in equation (9) is substantial in the corona [Krauss-Varban and Welsch, 2007] but only marginal in the solar wind and magnetosphere. In the case of electron heating it was suggested that the harder energetic particle spectra inferred from solar observations were also a consequence of the different values of $\beta$ in the corona versus the magnetosphere: particle acceleration in a finite $\beta$ plasma is constrained as energetic particles approach the marginal fire hose condition [Drake et al., 2006]. In any case in the present paper we do not attempt to address the production of high-energy particles during reconnection.

\section{Appendix A: Ion Bounce Frequency in the Magnetic Field Reversal Region}

[39] The Speiser-like orbit seen in Figure 4 is a consequence of the separation between the bounce frequency of particles across the reversal region and the slow gyration of particles in the normal magnetic field $B_{y}$ that threads the reversal region $\Omega_{0 y}$ [Büchner and Zelenyi, 1986; Chen and Palmadesso, 1986; Büchner and Zelenyi, 1989]. The bounce frequency $\omega_{b H}$ differs from that in a simple reversed field $\omega_{b}$ because of the presence of the Hall magnetic field. We can calculate this bounce frequency analytically for the simple magnetic field model in equation (3) in the limit where $L_{H} \rightarrow 0$ and where $L$ is large enough so that $B_{0 x} \simeq$ $B_{0 x}^{\prime} y$. In the deHoffman-Teller frame $\mathbf{E}=\mathbf{0}$. We consider for simplicity a particle with $\mathbf{v}=\left(0, v_{0}, 0\right)$ at $y=0$. The magnetic field can be written in terms of the vector potential $\mathbf{A}=\left(-B_{0 z}|y|, 0, B_{0 x}^{\prime} y^{2} / 2\right)$. Using the conservation of canonical momentum in the $x$ and $z$ directions, we find

$$
\begin{aligned}
& v_{x}=\frac{1}{m_{p}}\left(p_{x}-\frac{e A_{x}}{c}\right)=-\frac{e A_{x}}{m_{p} c}, \\
& v_{z}=\frac{1}{m_{p}}\left(p_{z}-\frac{e A_{z}}{c}\right)=-\frac{e A_{z}}{m_{p} c} .
\end{aligned}
$$

The conservation of energy then yields

$$
v_{y}^{2}+\Omega_{0 z}^{2} y^{2}+\frac{\Omega_{0 x}^{2}}{4 L^{2}} y^{4}=v_{0}^{2} .
$$

The terms quadratic and quartic in $y$ guarantee that the particle will have a maximum excursion $\Delta$ in $y$ given by

$$
\Delta^{2}=\frac{2 v_{0}^{2}}{\omega_{b}^{4}}\left(\sqrt{\Omega_{0 z}^{4}+\omega_{b}^{4}}-\Omega_{0 z}^{2}\right)
$$


The characteristic bounce frequency defined by $\omega_{b H} \equiv v_{0} / \Delta$ is then given in equation (5). We note that $\omega_{b H}$ is the actual particle bounce frequency only in the limit of $\Omega_{0 z}$ large. For other parameters it differs from the actual bounce frequency by factors of order unity.

[40] Acknowledgments. This work was supported in part by NSF grants ATM0613782 and ATM0752503 and NASA grants NNG06GH23G and NNX07AU92G. Thank you to S. Eriksson for input on the ACE data.

[41] Amitava Bhattacharjee thanks the reviewers for their assistance in evaluating this paper.

\section{References}

Arzner, K., and M. Scholer (2001), Kinetic structure of the post plasmoid plasma sheet during magnetotail reconnection, J. Geophys. Res., 106, 3827.

Büchner, J., and L. M. Zelenyi (1986), Deterministic chaos in the dynamics of charged particles near a magnetic field reversal, Phys. Lett. A, 118, 395.

Büchner, J., and L. M. Zelenyi (1989), Regular and chaotic charged particle motion in magnetotaillike field reversals: 1 . Basic theory of trapped motion, J. Geophys. Res., 94(11), 821.

Chen, J., and P. J. Palmadesso (1986), Chaos and nonlinear dynamics of single-particle orbits in a magnetotaillike magnetic field, J. Geophys. Res., 91, 1499.

Chen, L.-J., et al. (2008), Observation of energetic electrons within magnetic islands, Nat. Phys., 4, 19.

Coroniti, F. V. (1971), Laminar wave-train structure of collisionless magnetic slow shocks, Nucl. Fusion, 11, 261.

Cowley, S. W. H., and P. Shull Jr. (1983), Current sheet acceleration of ions in the geomagnetic tail and the properties of ion bursts observed at the lunar distance, Planet. Space Sci., 31, 235.

Cranmer, S. R., G. B. Field, and J. L. Kohl (1999), Spectroscopic constraints on models of ion cyclotron resonance heating in the polar solar corona and high-speed solar wind, Astrophys. J., 518, 937.

Drake, J. F., H. Che, M. A. Shay, and M. Swisdak (2006), Electron acceleration from contracting magnetic islands during reconnection, Nature, $443,553$.

Drake, J. F., M. A. Shay, and M. Swisdak (2008), The Hall fields and fast magnetic reconnection, Phys. Plasmas, 15, 042306.

Emslie, A. G., et al. (2004), Energy partition in two solar flare/CME events, J. Geophys. Res., 109, A10104, doi:10.1029/2004JA010571.

Gosling, J. T. (2007), Observations of magnetic reconnection in the turbulent high-speed solar wind, Astrophys. J., 671, L73.

Gosling, J. T., M. F. Thomsen, S. J. Bame, and C. T. Russell (1986), Accelerated plasma flows at the near-tail magnetopause, J. Geophys. Res., 91, 3029.

Gosling, J. T., R. M. Skoug, D. K. Haggerty, and D. J. McComas (2005a), Absence of energetic particle effects associated with magnetic reconnection exhausts in the solar wind, Geophys. Res. Lett., 32, L14113, doi:10.1029/2005GL023357.

Gosling, J. T., R. M. Skoug, D. J. McComas, and C. W. Smith (2005b), Direct evidence for magnetic reconnection in the solar wind near $1 \mathrm{AU}$, J. Geophys. Res., 110, A01107, doi:10.1029/2004JA010809.

Gosling, J. T., S. Eriksson, R. M. Skoug, D. J. McComas, and R. J. Forsyth (2006), Petschek-type reconnection exhausts in the solar wind well beyond 1 AU: Ulysses, Astrophys. J., 644, 613.

Gosling, J. T., S. Eriksson, T. D. Phan, D. E. Larson, R. M. Skoug, and D. J. McComas (2007), Direct evidence for prolonged magnetic reconnection at a continuous x-line within the heliospheric current sheet, Geophys. Res. Lett., 34, L06102, doi:10.1029/2006GL029033.

Hoshino, M., T. Mukai, and T. Yamamoto (1998), Ion dynamics in magnetic reconnection: Comparison between numerical simulation and geotail observations, J. Geophys. Res., 103, 4509.

Imada, S., R. Nakamura, P. W. Daly, M. Hoshino, W. Baumjohann, S. Mühlbachler, A. Balogh, and H. Rème (2007), Energetic electron acceleration in the downstream reconnection outflow region, J. Geophys. Res., 112, A03202, doi:10.1029/2006JA011847.

Karimabadi, H., D. Krauss-Varban, N. Omidi, and H. X. Vu (1999), Magnetic structure of the reconnection layer and core field generation in plasmoids, J. Geophys. Res., 104, 12,313.

Kliem, B. (1994), Particle orbits, trapping and acceleration in a filamentary current sheet model, Astrophys. J., 90, 719.

Krauss-Varban, D., and N. Omidi (1995), Large-scale hybrid simulations of the magnetotail during reconnection, Geophys. Res. Lett., 22, 3271.
Krauss-Varban, D., and B. T. Welsch (2007), Solar flare particle heating via low- $\beta$ reconnection, in Proceedings of the International Astronomical Union, vol. 2, Highlights of Astronomy, vol. 14, edited by K. A. van der Hucht, 89 pp., Cambridge Univ. Press, Cambridge, U. K.

Lin, R. P., et al. (2003), RHESSI observations of particle acceleration and energy release in an intense solar gamma-ray line flare, Astrophys. J., 595, L69.

Mason, G. M. (2007), ${ }^{3} \mathrm{He}$-rich solar energetic particle events, Space Sci. Rev., 130, 231.

Matthaeus, W. H., J. J. Ambrosiano, and M. J. Goldstein (1984), Particle acceleration by turbulent magnetohydrodynamic reconnection, Phys. Rev. Lett., 53, 1449.

Meng, C.-I., A. T. Y. Lui, S. M. Krimigis, and S. Ismail (1981), Spatial distribution of energetic particles in the distant magnetotail, J. Geophys. Res., 86, 5682.

Miller, J. A. (1998), Particle acceleration in impulsive solar flares, Space Sci. Rev., 86, 79 .

Möbius, E., D. Hovestadt, B. Klecker, M. Scholer, G. Gloeckler, and F. M. Ipavich (1985), Direct observation of $\mathrm{He}^{+}$pick-up ions of interstellar origin in the soloar wind, Nature, 318, 426.

Øieroset, M., R. P. Lin, T. D. Phan, D. E. Larson, and S. D. Bale (2002), Evidence for electron acceleration up to $\sim 300 \mathrm{keV}$ in the magnetic reconnection diffusion region of the Earth's magnetotail, Phys. Rev. Lett., 89,195001

Onofri, M., H. Isliker, and L. Vlahos (2006), Stochastic acceleration in turbulent electric fields generated by $3 \mathrm{D}$ reconnection, Phys. Rev. Lett., 96, 151102

Petrosian, V., and S. Liu (2004), Stochastic acceleration of electrons and protons. I. Acceleration by parallel-propagating waves, Astrophys. J., $610,550$.

Petschek, H. E. (1964), Magnetic field annihilation, in AAS-NASA Symposium on the Physics of Solar Flares, edited by W. N. Hess, NASA Spec. Publ., vol. 50, p. 425.

Phan, T. D., et al. (2006), A magnetic reconnection $\mathrm{x}$-line extending more than 390 Earth radii in the solar wind, Nature, 439, 175 .

Phan, T. D., J. F. Drake, M. A. Shay, F. S. Mozer, and J. P. Eastwood (2007a), Evidence for an elongated ( $>60$ ion skin depths) electron diffusion region during fast magnetic reconnection, Phys. Rev. Lett., 99, 255002.

Phan, T. D., G. Paschmann, C. Twitty, F. S. Mozer, J. T. Gosling, J. P. Eastwood, M. Øieroset, H. Rème, and E. A. Lucek (2007b), Evidence for magnetic reconnection initiated in the magnetosheath, Geophys. Res. Lett., 34, L14104, doi:10.1029/2007GL030343.

Sarafopoulos, D. V., N. F. Sidiropoulos, and E. T. Sarris (2001), The dawndusk plasma sheet asymmetry of energetic particles: An interball perspective, J. Geophys. Res., 106, 13,053.

Shay, M. A., J. F. Drake, and M. Swisdak (2007), Two-scale structure of the electron dissipation region during collisionless magnetic reconnection, Phys. Rev. Lett., 99, 155002.

Shibata, K., and S. Tanuma (2001), Plasmoid-induced-reconnection and fractal reconnection, Earth Planets Space, 53, 473.

Speiser, T. W. (1965), Particle trajectories in a model current sheet, based on the open model of the magnetosphere, with applications to auroral particles, J. Geophys. Res., 70, 1717.

Tsuneta, S. (1996), Structure and dynamics of magnetic reconnection in a solar flare, Astrophys. J., 456, 840 .

von Steiger, R., and T. H. Zurbuchen (2006), Kinetic properties of heavy solar wind ions from Ulysses-SWICS, Geophys. Res. Lett., 33, L09103, doi:10.1029/2005GL024998.

Wygant, J. R., et al. (2005), Cluster observations of an intense normal component of the electric field at a thin reconnecting current sheet in the tail and its role in the shock-like acceleration of the ion fluid into the separatrix region, J. Geophys. Res., 110, A09206, doi:10.1029/ 2004JA010708.

Yin, L., D. Winske, W. Daughton, and F. V. Coroniti (2005), Dissipation in oblique slow shocks, J. Geophys. Res., 110, A09217, doi:10.1029 2005JA011031.

Yin, L., D. Winske, and W. Daughton (2007), Kinetic Alfvén waves and electron physics. II. Oblique slow shocks, Phys. Plasmas, 14, 062105.

Zeiler, A., D. Biskamp, J. F. Drake, B. N. Rogers, M. A. Shay, and M. Scholer (2002), Three-dimensional particle simulations of collisionless magnetic reconnection, J. Geophys. Res., 107(A9), 1230, doi:10.1029/ 2001JA000287.

P. A. Cassak, Department of Physics, West Virginia University, Morgantown, WV 26506, USA. (paul.cassak@mail.wvu.edu)

J. F. Drake and M. Swisdak, IREAP, University of Maryland, College Park, MD 20742, USA. (drake@umd.edu; swisdak@umd.edu) 
S. T. Lepri and T. H. Zurbuchen, Department of Atmospheric, Oceanic, and Space Sciences, University of Michigan, Ann Arbor, MI, USA. (slepri@umich.edu; thomasz@umich.edu)

R. P. Lin and T. D. Phan, Space Sciences Laboratory, University of California, Berkeley, CA 94720, USA. (rlin@ssl.berkeley.edu; phan@ssl. berkeley.edu)
E. Quataert, Department of Astronomy, University of California Berkeley, CA 94720, USA. (eliot@astro.berkeley.edu)

M. A. Shay, Department of Physics and Astronomy, University of Delaware, Newark, DE 19716, USA. (shay@physics.udel.edu) 